\title{
Complexity Results for Argumentation-based Agent Communication
}

\author{
Jamal Bentahar ${ }^{1}$ and Zakaria Maamar ${ }^{2}$ \\ ${ }^{1}$ CIISE, Concordia University, Montreal, Canada bentahar@ ciise.concordia.ca \\ ${ }^{2}$ CIT, Zayed University, Dubai, U.A.E zakaria.maamar@zu.ac.ae
}

\begin{abstract}
In autonomous multi-agent systems, agents communicate with each other in order to cooperatively resolve problems and achieve joint goals. In advanced applications such as ebusiness, agents should be able to communicate not only by exchanging simple massages, but by engaging in complex conversations such as persuasions and negotiations. In this paper, we address the computational complexity problem of such conversations where agents are equipped with reasoning capabilities based upon argumentation. We argue that the computational efficiency of these conversations depends on the underlying logical language and on the type of reasoning mechanisms. In addition, we show how a trade-off between complexity and flexibility could be achieved.
\end{abstract}

Key Words. Agent Computing, Agent Communication, Argumentation, Reasoning, Computational Complexity.

\section{Introduction}

One of the important characteristics of agent-based computing is the ability of agents to communicate. For the requirements of advanced applications such as e-business, emarketplace, and virtual organizations in grid-computing, this communication should go beyond the simple message exchange. Agents should be able to find compromises on resources through negotiation, persuade each other to adopt some solutions, influence each other to make some decisions, etc. [16]. They need advanced reasoning and decision making capabilities allowing them to build arguments so that they can justify their choices and influence other agents' decisions [12]. This type of reasoning is based on argumentation and has been extensively investigated these last few years within the multi-agent community $[2,7]$.

In artificial intelligence, argumentation is defined as a process that supports the exchange of arguments that either back or refute some conclusion [15]. An important branch of argumentation is formal dialectics [9, 11]. In its most abstract form, a dialectical model is a set of arguments and a binary relation representing the attack-relation (and indirectly the defense relation) between the arguments in a dialogue. Dialectical models are relevant for automated negotiation, in which agents should persuade each other. A single agent may use such an argumentation to perform its reasoning because it needs to make decisions in highly dynamic environments, considering interacting preferences and utilities. In addition, this argumentation can help multiple agents to interact rationally, by giving and receiving reasons for conclusions and decisions.

Although many argumentation frameworks for multiagent systems and agent communication have been proposed $[3,6,7]$, only few works have addressed the problem of assessing their computational complexity [5, 14]. The reason is that these frameworks have been proposed, not to be computationally efficient, but to achieve more flexibility and to equip agents with powerful reasoning capabilities, which increases their complexity. The purpose of this paper is to investigate this issue and to determine the important factors contributing to this complexity. This is of great importance to chose the techniques to be used when implementing concrete applications such as agent-based Web services and agent-oriented e-applications. We argue that the computational efficiency of argumentation-based agent communication depends on both the logical language that agents use to express and reason about their knowledge, and the flexibility and strongness of this reasoning. Finally, we show how a trade-off between complexity and flexibility could be achieved.

This paper is organized as follows. Section 2 presents the analyzed agent communication framework in terms of concepts and argumentation-based reasoning. Section 3 discusses the computational complexity of this framework. Section 4 concludes the paper and identifies some ideas for future work.

\section{A Framework for Agent Communication}

\subsection{Argumentative Reasoning}

In many agent communication proposals, the agent architecture is mainly composed of three layers: mental, so- 
cial, and reasoning $[1,3]$. The mental layer includes beliefs, desires, goals, etc. The social layer captures social concepts such as norms, protocols, roles, etc. Agents use their reasoning capabilities to reason about their private mental states and about the public notions. For example, agents reason about their current beliefs and the shared communication protocol to decide about the next communicative act to perform. An agent's reasoning capabilities are represented by the reasoning layer using an argumentation system. We distinguish between internal and external arguments. The former are used to manage the incoherences of the agent's beliefs and the latter are used to manage inconsistencies between an agent's beliefs and information conveyed by an addressee. All the communicative acts that agents perform are supported by arguments. For example, in a negotiation setting, agent $A g_{1}$ accepts an addressee's proposal if $A g_{1}$ can build an argument out of its knowledge base in favor of the proposal. If a counter-argument can be built out of this knowledge base, $A g_{1}$ refuses the proposal and makes a counter-proposal.

Formally, an argumentation system consists of a logical language $£$ used to represent agents' knowledge, and definitions of the notions of argument, attack relation between arguments, and acceptability [9]. Let us introduce these definitions where $\vdash$ stands for classical inference.

Definition 1 (Argument). Let $\Gamma$ be a non-necessarily consistent knowledge base with no deductive closure. An argument is a pair $(H, h)$ where $h$ is a formula in $£$ and $H$ a subset of $\Gamma$ such that (i) $H$ is consistent and (ii) $H \vdash h$. The set $H$ is called the support of the argument and $h$ its conclusion.

Definition 2 (Minimal Argument). Let $(H, h)$ be an argument. $(H, h)$ is minimal if there is no proper subset $H^{\prime}$ of $H\left(H^{\prime} \subset H\right)$ such that $\left(H^{\prime}, h\right)$ is an argument.

Because agents' knowledge bases are not necessarily consistent, they should be able to reason about their preferences in relation to beliefs. The objective is to state that some facts are more strongly believed than others. Hence, we assume that any set of facts has a preference order over it. This ordering exists because an agent's knowledge base denoted by $\Gamma$ is stratified into non-overlapping sets $\Gamma_{1}, \ldots, \Gamma_{n}$ such that facts in $\Gamma_{i}$ are all equally preferred and are more preferred than those in $\Gamma_{j}$ where $i<j$. We define the preference level of a subset of $\Gamma$ whose elements belong to different non-overlapping sets as follows.

Definition 3 (Preference Level). The preference level of a nonempty subset $\gamma$ of $\Gamma$ denoted by level $(\gamma)$ is the number of the highest numbered layer which has a member in $\gamma$.

Example 1. Let $\Gamma=\Gamma_{1} \cup \Gamma_{2}$ with $\Gamma_{1}=\{a, b\}$ and $\Gamma_{2}=$ $\{c, d\}$ and $\gamma=\{a\}$ and $\gamma^{\prime}=\{a, d\}$. We have: level $(\gamma)=$ 1 (because $\left.a \in \Gamma_{1}\right)$ and level $\left(\gamma^{\prime}\right)=2$ (because $\left.d \in \Gamma_{2}\right)$.
Definition 4 (Preference Relation). Let $(H, h)$ and $\left(H^{\prime}, h^{\prime}\right)$ be two minimal arguments. $(H, h)$ is preferred to $\left(H^{\prime}, h^{\prime}\right)$ (denoted by $\left.(H, h) \succ_{\text {pref }}\left(H^{\prime}, h^{\prime}\right)\right)$ iff level $(H)<$ level $\left(H^{\prime}\right)$.

Definition 5 (Attack Relation). Let $(H, h)$ and $\left(H^{\prime}, h^{\prime}\right)$ be two minimal arguments. $(H, h)$ attacks $\left(H^{\prime}, h^{\prime}\right)$ (denoted by $\left.(H, h) \mathcal{A T}\left(H^{\prime}, h^{\prime}\right)\right)$ iff $\left(H \vdash \neg h^{\prime}\right.$ or $\exists x^{\prime} \in H^{\prime}$ : $\left.H \vdash \neg x^{\prime}\right)$ and $(H, h) \succ$ pref $\left(H^{\prime}, h^{\prime}\right)$ where “ $\neg$ " represents strict negation. In other words, an argument is attacked if and only if there exists a preferred argument that strictly negates its conclusion or one of its premises.

Definition 6 (Argumentation System). It is a pair $\langle\mathcal{A}, \mathcal{A T}\rangle$, where $\mathcal{A}$ is a set of arguments and $\mathcal{A T} \subseteq \mathcal{A} \times \mathcal{A}$ is the attack relation. For a subset $S$ of arguments in the argumentation system $\langle\mathcal{A}, \mathcal{A T}\rangle$, it is said that:

1. $a \in \mathcal{A}$ is acceptable with respect to $S$ if $\forall b \in \mathcal{A}$ s.t. $b \mathcal{A T} a, \exists c \in S$ s.t. $c \mathcal{A} \mathcal{A}$ b.

2. $S$ is conflict-free if no argument in $S$ is attacked by any other argument in $S$.

\subsection{Strategic Reasoning}

Argumentative reasoning allows agents to build arguments that support their beliefs and choices or attacking addressees's arguments in order to achieve a consensus in a persuasion or negotiation setting. However, this reasoning does not specify how agents can select an argument out of several ones. The reasoning mechanism enabling agents to decide about the best argument to use is called strategic reasoning [4]. The idea is to be able to use the arguments having the best chance to be accepted by the addressee. For example, if $A g_{1}$ knows that addressee $A g_{2}$ does not like ideological ideas from religion, $A g_{1}$ will avoid arguments related to religion and use rather another type of arguments. Consequently, agents should consider not only their own beliefs, but also these addressees' beliefs and preferences. In this context, a strategy is defined as a function $\mathcal{S} t r$ mapping a set of possible arguments to an argument (the best one). Let $\mathcal{A}$ be the set of all arguments. This function is defined as follows:

$$
\text { Str }: 2^{\mathcal{A}} \rightarrow \mathcal{A} \text {. }
$$

This reasoning requires building all acceptable arguments (Definition 6) and ordering them according to the agent's beliefs about the addressee's preferences. We suppose that each fact $\alpha$ in the agent's knowledge base has an acceptance chance value $A c c(\alpha)_{A g_{j}}$ that reflects the probability that the addressee $A g_{j}$ will accept it. We define the acceptance chance of a subset $\gamma$ of the knowledge base as follows:

$$
A c c(\gamma)_{A g_{j}}=\min _{\alpha \in \gamma}\left(A c c(\alpha)_{A g_{j}}\right)
$$


Arguments can be compared according to the Better-than relation defined as follows:

Definition 7 (Better-than Relation). Let $(H, h)$ and $\left(H^{\prime}, h^{\prime}\right)$ be two acceptable arguments. $(H, h)$ is better than $\left(H^{\prime}, h^{\prime}\right)$ according to an agent's beliefs about the addressee's $A g_{j}$ preferences iff $A c c(H)_{A g_{3}} \geq A c c\left(H^{\prime}\right)_{A g_{j}}$. This relation is denoted by $(H, h) \succeq$ bet $\left(H^{\prime}, h^{\prime}\right)$.

\section{Computational Complexity}

\subsection{Logical Language}

To use argumentative reasoning in their conversations, agents should build arguments from their knowledge bases, defend them, and attack addressee's arguments. The first factor in the computational complexity of such a reasoning is the language used to build these arguments. The second factor is the way the agents' knowledge bases are maintained (are always consistent, or could be inconsistent). The third factor is the reasoning type itself: agents build any argument to support or attack a fact, or rather they build "good" arguments.

Before discussing these factors, let us introduce the polynomial time hierarchy used in the complexity theory $[10,13]$ :

$$
\Delta_{0}^{p}=\sum_{0}^{p}=\prod_{0}^{p}=\mathrm{P} \text { (class of problems that can be solved }
$$

$$
\text { and } \begin{aligned}
\forall k \geq 0, \Delta_{k+1}^{p} & =\mathrm{P}^{\sum_{k}^{p}}, \sum_{k+1}^{p}=\mathrm{NP}^{\sum_{k}^{p}}, \\
\prod_{k+1}^{p} & =\operatorname{co}-\sum_{k+1}^{p}
\end{aligned}
$$

In particular:

$$
\begin{gathered}
\mathrm{NP}=\sum_{1}^{p}, \text { co- } \mathrm{NP}=\prod_{1}^{p}, \mathrm{NP}^{\mathrm{NP}}=\sum_{2}^{p}, \\
\mathrm{co-NP} \mathrm{NP}=\prod_{2}^{p}, \text { and } \Delta_{2}^{p}=\mathrm{P}^{\mathrm{NP}} .
\end{gathered}
$$

Only the problems in $\Delta_{0}^{p}$ and $\Delta_{1}^{p}$ are tractable (could be solved in a polynomial time). $\Delta_{1}^{p}=\mathrm{P}^{\mathrm{P}}$ is the class of problems that are solved in polynomial time with independent queries (or calls) to an $\mathrm{P}$ oracle. $\sum_{1}^{p}$ is simply the class of problems solved in non-deterministic polynomial time. $\prod_{1}^{p}$ is the class of problems whose dual are NP. $\sum_{2}^{p}$ is the class of problems that are solved in NP time with independent queries to a NP oracle and $\prod_{2}^{p}$ is its dual. Because building proofs in the first-order logic is semi-decidable, we have the following direct result.

Proposition 1. Building an argument for a formula $h$ from a non-consistent and first-order logic knowledge base is semi-decidable.

Consequently, although the first-order logic has a powerful expressiveness, it can not be used to implement argumentation-based systems for agent communication. Let us now consider the case of the propositional logic.
Theorem 1. Given an inconsistent and propositional knowledge base $\Gamma$, and a formula $h$. Deciding whether there is an argument for $h$ from $\Gamma$ is $\sum_{2}^{p}$-complete.

Proof. Let us first prove the membership to $\sum_{2}^{p}$. The following algorithm resolves the problem:

1- Guess a subset $H$ of $\Gamma$.

2- Check if $(H, h)$ is an argument.

To check if $(H, h)$ is an argument, we need to check that $H$ is consistent (satisfiability problem), and that $H \vdash h$ (validity problem). Because satisfiability is NP-Complete and validity is co-NP-complete, the problem is clearly in $\Delta_{2}^{P}$, hence membership in $\sum_{2}^{p}$ follows.

To prove the completeness, we use a transformation from deciding the validity of a Quantified Boolean Formula with two quantifier alternations ( $\exists$ and $\forall)\left(Q B F_{2, \exists}\right.$ ) [10]. Let us without loss of generality consider the following $Q B F_{2, \exists}$ $\Phi$ formula: $\Phi=\exists x_{1}, \ldots, x_{n} \forall y_{1}, \ldots, y_{m} h$. We show that $\Phi$ is valid iff $\exists H:(H, h)$ is an argument. Let us suppose that $\Phi$ is valid. We define $H$ as a set of formulas over $x_{1}, \ldots, x_{n}$. It is clear that $H$ is consistent and $H \vdash h$. Consequently $(\mathrm{H}, \mathrm{h})$ is an argument. Let us now suppose that $\exists H:(H, h)$ is an argument, and show that $\Phi$ is valid. Consequently, $H \rightarrow h$ is a tautology. Because $H$ is consistent and expressed over $x_{1}, \ldots, x_{n}$, the validity of $\Phi$ is obtained.

Without considering the problem of minimality, Theorem 1 shows that argumentative reasoning is intractable in the propositional case. Consequently, although it is suitable to express knowledge in different fields such as e-business and service-based computing, propositional logic is not appropriate to implement agent communication for concrete applications.

Let us now consider the case of propositional Horn clauses which are a simpler version of the propositional logic. A propositional Horn clause is a disjunction of literals with at most one positive literal $\neg p_{1} \vee \neg p_{2} \vee \ldots \vee \neg p_{n} \vee c$ (also written as implication $p_{1} \wedge p_{2} \wedge \ldots \wedge p_{n} \rightarrow c$ ). A propositional Horn formula is a conjunction of propositional Horn clauses. These clauses could be restricted to be definite where each clause has exactly one positive literal. A propositional definite Horn formula is a conjunction of propositional definite Horn clauses. This restriction is of a particular interest in modeling argumentative reasoning, since formulas of type $p_{1} \wedge p_{2} \wedge \ldots \wedge p_{n} \rightarrow c$ are adequate to describe interrelationships between premises and conclusions. This could be used to support positive literals.

Proposition 2. Given a Horn knowledge base $\Gamma$, a subset $H \subseteq \Gamma$, and a formula $h$. Checking whether $(H, h)$ is an argument is polynomial.

Proof. From the linear time algorithms for Horn satisfiability in [8], it follows that the Horn implication problem 
$H \vdash h$ is decidable in $O(|H| \times|h|)$ time. From the same result, it also follows that deciding whether $H$ is consistent is polynomial.

Proposition 3. Given a Horn knowledge base $\Gamma$, and an argument $(H, h)$ over $\Gamma$. Checking whether $(H, h)$ is minimal is polynomial.

Proof. Let $l$ be a literal. The following algorithm resolves the problem:

$\forall l \in H$ check if $H-\{l\} \vdash h$. Because the implication problem is polynomial, we are done.

Consequently, unlike propositional logic, minimality is not an additional source of complexity in the case of Horn clauses. The problem is linear with the size of $H$.

Theorem 2. Given a Horn knowledge base $\Gamma$ and a formula $h$, deciding whether there is an argument $(H, h)$ is NP-complete.

Proof. Membership of this problem in NP is straightforward since by Proposition 2, checking that a guessed subset $H \subseteq \Gamma$ is consistent and $H \vdash h$ could be done in a polynomial time.

To prove the hardness of the problem, we use a transformation from the well-known SAT problem. Let $C=$ $\left\{C_{1}, C_{2}, \ldots, C_{n}\right\}$ be a set of propositional clauses on $X=$ $\left\{x_{1}, x_{2}, \ldots, x_{n}\right\}$, and let $X^{\prime}=\left\{x_{1}^{\prime}, x_{2}^{\prime}, \ldots, x_{n}^{\prime}\right\}, Y=$ $\left\{y_{1}, y_{2}, \ldots, y_{m}\right\}$ be sets of new variables. Then, there is an argument $(H, h)$ over $\Gamma$ where $h$ is a formula over $Y$ and

$$
\begin{gathered}
\Gamma=X \cup X^{\prime} \cup\left\{\neg x_{i} \vee \neg x_{i}^{\prime}: 1 \leq i \leq n\right\} \cup \\
\bigcup_{i=1}^{m}\left(\left\{x_{j} \rightarrow y_{i}: x_{j} \in C_{i}\right\} \cup\left\{x_{j}^{\prime} \rightarrow y_{i}: \neg x_{j} \in C_{i}\right\}\right),
\end{gathered}
$$

iff $C$ is satisfiable. Since $\Gamma$ is constructible in polynomial time, we are done.

This theorem shows that using general Horn clauses also results on intractable reasoning procedures for argumentation-based agent communication. However, this complexity is less than the one associated with the fully propositional logic. For relatively small knowledge bases, argumentative reasoning based upon Horn clauses is possibly implementable.

Let us now consider the case of definite Horn clauses.

Proposition 4. Let $\Gamma$ be a definite Horn knowledge base, $h$ a formula, and $\mathcal{A}$ the set of arguments over $\Gamma$.

$\exists H \subseteq \Gamma:(H, h) \in \mathcal{A} \Rightarrow \forall H^{\prime}: H \subseteq H^{\prime} \subseteq \Gamma,\left(H^{\prime}, h\right) \in$ $\mathcal{A}$.

Proof. If $(H, h)$ is an argument where $H$ is a set of definite Horn formulas under the form $c$ or $p_{1} \wedge p_{2} \wedge \ldots \wedge p_{n} \rightarrow c$ where $p_{1}, p_{2}, \ldots, p_{n}, c$ are positive literals, then adding any definite Horn formula to $H$ will result in a consistent set of formulas $H^{\prime}: \Gamma \supseteq H^{\prime} \supseteq H$. Since $H \vdash h$, it follows that $H^{\prime} \vdash h$, whence the proposition.

Theorem 3. Given a definite Horn knowledge base $\Gamma$ and a formula $h$. Deciding whether there is an argument $(H, h)$ is polynomial.

Proof. From Proposition 4, it follows that there is an argument supporting $h$ iff $(\Gamma, h) \in \mathcal{A}$. Because every definite Horn knowledge base is a Horn knowledge base, then by Proposition 2, the theorem follows.

This result shows that building arguments is tractable when knowledge is represented by definite Horn clauses. Now, let us discuss the complexity of the attack relation in this particular case. To simplify the problem, instead of considering two knowledge bases $\Gamma_{1}$ for $A g_{1}$ and $\Gamma_{2}$ for $A g_{2}$, we consider, without loss of generality, a single knowledge base $\left(\Gamma=\Gamma_{1} \cup \Gamma_{2}\right)$. The results are the same since the union of the two knowledge bases could be inconsistent. The following proposition is straightforward.

Proposition 5. Let $\Gamma$ be a definite Horn knowledge base and $(H, h)$ and $\left(H^{\prime} . h^{\prime}\right)$ be two arguments over $\Gamma$. Deciding whether $(H, h) \succ_{\text {pref }}\left(H^{\prime}, h^{\prime}\right)$ is polynomial.

Proposition 6. Let $\Gamma$ be a definite Horn knowledge base and $(H, h)$ and $\left(H^{\prime} . h^{\prime}\right)$ be two arguments over $\Gamma$. Deciding whether $(H, h) \mathcal{A T}\left(H^{\prime}, h^{\prime}\right)$ is polynomial.

Proof. The first part of the first condition of Definition 5 $\left(H \vdash \neg h^{\prime}\right)$ is polynomial. The second part $\left(\exists x^{\prime} \in H^{\prime}\right.$ : $\left.H \vdash \neg x^{\prime}\right)$ is decidable in $O\left(|H| \times\left|H^{\prime}\right| \times\left|\neg x^{\prime}\right|\right)$, and the second condition $\left((H, h) \succ_{\text {pref }}\left(H^{\prime}, h^{\prime}\right)\right)$ is polynomial by Proposition 5, whence the proposition.

Theorem 4. Let $\Gamma$ be a definite Horn knowledge base and $(H, h)$ an argument over $\Gamma$. Deciding whether there is an attacker of $(H, h)$ over $\Gamma$ is polynomial.

Proof. Since the preference check is polynomial (from Proposition 5), building an argument attacking a given argument has the same complexity than building an argument supporting a conclusion. From Theorem 3 we are done.

These results prove that Definite Horn logic-based argumentative reasoning is tractable.

\subsection{Knowledge Base and Reasoning Types}

Having discussed the first factor: the adopted logical language, we consider in this section the other two factors: the structure of the knowledge base (consistent or not), and the reasoning type (strategic or not). In the case of propositional logic, the fact that the knowledge base is consistent 
reduces the complexity since there is no need to check the consistency, which is NP-complete. However, this restriction does not make the problem tractable since $\Gamma \vdash h$ is co-NP-complete. In the case of definite Horn logic, the tractability is due to the guarantee that the knowledge base is consistent. Consequently we have the following corollaries from Theorems 3 and 4 .

Corollary 1. Given a consistent Horn knowledge base $\Gamma$ and a formula $h$. Deciding whether there is an argument $(H, h)$ over $\Gamma$ is polynomial.

Corollary 2. Let $\Gamma$ be a consistent Horn knowledge base and $(H, h)$ an argument over $\Gamma$. Deciding whether there is an attacker of $(H, h)$ over $\Gamma$ is polynomial.

All the results obtained above consider only the argumentative reasoning. Let us now discuss the strategic reasoning. Because the problem is intuitively more demanding, it is clear that the reasoning procedure is intractable if the knowledge is expressed in the propositional logic. In the case of Horn clauses, the problem is also intractable if the knowledge base is not necessarily consistency (we can prove that the problem is $\mathrm{P}^{\mathrm{NP}[O(\log n)]}$-complete, where $n$ is the number of calls to an NP oracle). Finally, in the case of definite Horn clauses and consequently consistent Horn clauses, although the problem is more complex because agents should build all acceptable arguments, it is still tractable since checking the Better-than relation is clearly polynomial. We can easily prove the following theorem:

Theorem 5. Let $\Gamma$ be a consistent Horn knowledge base and $h$ a formula. Building a strategic argument for $h$ over $\Gamma$ is decidable in $O(|\Gamma|)$.

Strategic reasoning is then tractable (polynomial) under definite Horn clauses and, consequently, under the restriction of the Horn knowledge base consistency.

As a final result of this analysis, it is possible to achieve a trade-off between the flexibility of argumentation-based reasoning for agent communication and its computational complexity. The idea is, in one hand, using a restricted language such as definite Horn clauses, or imposing the consistency of the agents' knowledge bases whit the fully propositional Horn clauses, and, on the other hand, equipping agents with advanced and powerful strategic reasoning.

\section{Conclusion}

In this paper, we addressed the problem of computational complexity of communicating agents based on argumentation. We discussed the important factors that practitioners and developers should consider when implementing concrete applications using argumentative agent-computing. This analysis is of great importance for implementing these applications since the achievement of a desired trade-off between flexibility and complexity has been proved.

As future work, we intend to apply the argumentationbased framework to Web services and to discuss communication vs. computational trade-off. We also plan to investigate security issues in this context using argumentative agents and the technique of mechanism design.

\section{References}

[1] Amgoud L., Dimopoulos Y., and Moraitis P.: A Unified and General Framework for Argumentation-based Negotiation. Intern. Conference on Autonomous Agents and Multi-Agent Systems, USA, 2007.

[2] Atkinson, K., Bench-Capon, T., and McBurney, P.: A Dialogue Game Protocol for Multi-Agent Argument over Proposals for Action. Journal of AAMAS, 11(2), 2005, 153-171.

[3] Bentahar, J., Moulin, B., Meyer, J-J.Ch., and Lespérance, Y.: A New Logical Semantics for Agent Communication. In Computational Logic in Multi-Agent Systems, Springer LNAI 4371, 2006, 151-170.

[4] Bentahar, J., Mbarki, M., and Moulin, B.: Strategic and Tactic Reasoning for Communicating Agents. Argumentation in Multi-Agent Systems, Springer LNAI 4766, 2006, 142-160.

[5] Bentahar, J., Maamar, Z., Benslimane, D., and Thiran, Ph.: An Argumentation Framework for Communities of Web Services, IEEE Intelligent Systems, 22(6), 2007.

[6] Besnard, Ph. and Hunter, A.: A Logic-based Theory of Deductive Arguments. Artificial Intelligence 128, 2001, 203235.

[7] Chesñevar, C.I., Maguitman, A., and Loui, R.: Logical Models of Argument. ACM Computing Surveys 32, 2000, 337383.

[8] Dowling, W. and Gallier, J.H.: Linear-time Algorithms for Testing the Satisfiability of Propositional Horn Theories, Journal of Logic Programming, 1(3), 1984, 267-284.

[9] Dung, P.M., Kowalski, R.A., and Toni, F.: Dialectic Proof Procedures for Assumption-based, Admissible Argumentation. Artificial Intelligence 170(2), 2006, 114-159.

[10] Johnson, D.S.: A Catalog of Complexity Classes. Handbook of Theoretical Computer Science, Chapter 2. Elsevier Science Publishers, 1990.

[11] Kakas, A. and Moraitis, P.: Argumentation based Decision Making for Autonomous Agents. Intern. Conference on Autonomous Agents and Multi-Agent Systems, 2003, 883-890.

[12] McBurney, P., Parsons, S., and Wooldridge, M.: Desiderata for Agent Argumentation Protocols. Intern. Conference on AAMAS, 2002, 402-409.

[13] Papadimitriou, C.H.: Computational Complexity. Addison Wesley 1994.

[14] Parsons, S., Wooldridge, M., and Amgoud, L.: Properties and Complexity of Some Formal Inter-agent Dialogues. J. Log. Comput. 13(3), 2003, 347-376.

[15] Prakken, H. and Vreeswijk, G.: Logics for Defeasible Argumentation. Handbook of Philosophical Logic, 2000.

[16] Rahwan, I., Ramchurn,S.D., Jennings, N.R., McBurney, P., Parsons, S., and Sonenberg, L.: Argumentation-based Negotiation. Knowledge Engineering Review. 18(4), 2003, 343375. 